\title{
Fatores de risco para úlceras de pressão em idosos de Unidade de Terapia Intensiva
}

Recebido em: 08/12/2010

Aceito em : 14/04/2011

\author{
Marion Creutzberg ${ }^{1}$ \\ Nair Cristina Fortuna Aguilera² \\ Patrícia Cristina Cardoso ${ }^{3}$ \\ Thiago Lapuente Barbosa ${ }^{4}$ \\ Lieli Dapieve Ceolin 5 \\ Kátia Stein ${ }^{6}$ \\ Janete de Souza Urbanetto7
}

Objetivos: identificar o risco para úlceras de pressão (UP) em idosos de Unidade de Terapia Intensiva (UTI); comparar o risco de UP com as variáveis sexo, faixa etária e especialidade; comparar o risco de UP entre idosos, adultos jovens e médios. Método: estudo exploratório, analítico e retrospectivo realizado em uma UTI com amostra de 216 pacientes. Resultados: o risco de UP nas duas avaliações manteve-se elevado. Não houve diferença de risco de UP entre os pacientes idosos e os adultos jovens e médios. Conclusão: a avaliação do risco de UP é imprescindível para a qualidade da assistência em UTI.

Descritores: Úlcera por Pressão, Idoso, Unidades de Terapia Intensiva.

Risk factors for pressure ulcers in elderly patients in a Intensive Care Unit

Objectives: To identify risk of pressure ulcers (PU) on elderly in Intensive Care Unit (ICU), to compare the risk of PU with gender, age and specialty and to compare the risk of PU in the elderly, young and middle adults. Method: exploratory, analytical and retrospective in ICU with a sample of 216 patients. Results: the risk for PU in the two ratings remained high. There was no difference in risk for PU among the elderly and young and middle adults. Conclusion: the assessment of risk for PU is essential to the quality of ICU care.

Descriptors: Pressure Ulcer, Aged, Intensive Care Units.

\section{Los factores de riesgo para las úlceras por presión en ancianos en la Unidad de Cuidados Intensivos}

Objetivos: identificar el riesgo para las úlceras por presión (UPP) en la Unidad de Terapia Intensiva (UTI), para comparar el riesgo de la UPP con el sexo, la edad y la especialidad y para comparar el riesgo entre los adultos mayores, jóvenes y la mediana edad. Método: exploración, análisis y retrospectiva en la UTI con una muestra de 216 pacientes. Resultados: el riesgo de UPP en las dos clasificaciones se mantuvieron altos. No hubo diferencias en el riesgo de la UPP entre los adultos mayores y jóvenes y de mediana edad. Conclusión: la evaluación del riesgo de la UPP es esencial para la calidad de la atención en la UCI.

Descriptores: Úlcera por Presión, Anciano, Unidades de Cuidados Intensivos.

\section{INTRODUÇÃO}

$\mathrm{N}$ a última década, as instituições de saúde, os profissionais de saúde e a população em geral passaram a analisar as situações de risco existentes nos cenários de cuidado à saúde e a planejar e implementar ações para a prevenção de eventos adversos. A temática, embora já consolidada na literatura nacional e internacional, ainda encontra dificuldades para ser amplamente difundida nas instituições de saúde. A complexidade das organizações, o caráter multifatorial das situações que envolvem os eventos adversos e a sensibilidade que o tema desperta são aspectos que tornam difícil a percepção dos riscos e a implantação de uma abordagem de segurança ${ }^{(1,2)}$.

Entre os principais eventos adversos decorrentes da assistência à saúde inclui-se a úlcera de pressão (UP), uma lesão localizada na pele ou no tecido ou estrutura subjacente, geralmente sobre uma proeminência óssea, resultante de pressão isolada ou de pressão combinada com fricção e cisalhamento $^{(3)}$. A ocorrência de UP pode ocasionar desconforto físico para o paciente, aumento dos custos no tratamento, necessidade de cuidados intensivos, internação hospitalar prolongada, aumento do risco para complicações adicionais, necessidade de cirurgia corretiva e efeitos na taxa de mortalidade ${ }^{(4)}$.

O envelhecimento provoca alterações e desgastes em vários sistemas funcionais, que ocorrem de forma progressiva e irreversível(5). Em idades mais avançadas, as limitações visuais,

1 Enfermeira, Doutora em Gerontologia Biomédica. Professora Adjunta do curso de Enfermagem da Faenfi/PUCRS. Email: mcreutzberg@pucrs.br.

2 Enfermeira (PUCRS).

3 Enfermeira (PUCRS).

4 Enfermeiro (PUCRS).

5 Enfermeira Assistencial da UTI Geral do HSL/PUCRS

6 Enfermeira. Coordenadora Assistencial das Áreas de Intensivismo Adulto do HSL/PUCRS.

7 Enfermeira. Doutora em Ciências da Saúde, Professora Adjunta do curso de Enfermagem da Faenfi/PUCRS 
auditivas, motoras e intelectuais, bem como o surgimento de doenças crônico-degenerativas, intensificam-se, ocasionando a diminuição da condição de saúde ${ }^{(6)}$. Os pacientes idosos estão em maiores condições de risco para o agravamento do quadro inicial e, dessa forma, a probabilidade de desenvolvimento de UP eleva-se com o aumento da idade ${ }^{(7)}$. A pele do idoso apresenta alterações relacionadas com idade, genética, exposição solar, agentes químicos e tabagismo. A pele se torna seca, enrugada, flácida, com algumas neoplasias benignas e com maior predisposição à formação de bolhas traumáticas ${ }^{(8)}$.

O paciente em estado crítico tem grande tendência para formação de UP por fatores diversos, como sedação, alteração do nível de consciência, suporte ventilatório, uso de drogas vasoativas, restrição de movimentos por períodos prolongados e instabilidade hemodinâmica. Pela dificuldade na realização de medidas preventivas para a manutenção da integridade da pele ou pela gravidade do paciente, observa-se o aparecimento de UP em pacientes críticos hospitalizados ${ }^{(9)}$.

\section{OBJETIVOS}

O estudo teve como objetivos identificar o risco de UP em pacientes idosos internados em Unidade de Terapia Intensiva (UTI); comparar o risco de UP em idosos com as variáveis: sexo, faixa etária e especialidade; comparar o risco de UP entre idosos, adultos jovens e médios.

\section{MÉTODO}

Trata-se de um estudo exploratório analítico e retrospectivo(10).

A população do estudo foi composta de idosos internados na UTI de um hospital universitário, inseridos no Protocolo de Prevenção de Úlceras de Pressão e avaliados no período de janeiro a julho de 2010. A amostra foi composta de pacientes com idade de 21 anos ou mais, classificados como adultos jovens (22-39 anos), médios (40-59) e idosos (60 e mais) $)^{(11),}$ totalizando 216 pacientes. Embora o foco sejam os idosos, os pacientes não-idosos foram considerados sob o aspecto do risco de UP, a fim de atender ao objetivo de comparação de risco entre adultos idosos e adultos jovens e médios.

O Protocolo de Prevenção de UP avalia o risco por meio da escala de Braden e preconiza medidas de prevenção. A referida escala é um instrumento de avaliação constituído de seis itens que permitem quantificar o risco de desenvolver UP e determinar as medidas preventivas. Os itens avaliados são:

1. percepção sensorial, pontuada de 1 a 4: 1) totalmente limitado, 2) muito limitado, 3) levemente limitado ou 4) nenhuma limitação;

2. atividade física, pontuada de 1 a 4: 1) acamado, 2) confinado a cadeira, 3) caminha ocasionalmente ou 4) anda frequentemente;

3. umidade da pele, pontuada de 1 a 4: 1) completamente molhada, 2) muito molhada, 3) ocasionalmente molhada ou 4 raramente molhada;

4. mobilidade, pontuada de 1 a 4: 1) totalmente imóvel, 2) bastante limitado, 3) levemente limitado ou 4) não apresenta limitações;
5. nutrição, pontuada de 1 a 4:1) muito pobre, 2) provavelmente inadequada, 3) adequada ou 4) excelente;

6. fricção/cisalhamento, pontuada de 1 a 3: 1) problema, 2) problema potencial ou 3) nenhum problema ${ }^{(12)}$.

Depois de avaliadas cada uma das seis subescalas, somamse as respectivas pontuações e obtém-se uma pontuação total. Quanto menor o valor, maior a exposição ao risco. Os intervalos têm como significado: risco inexistente (21-23); risco baixo (1720); risco moderado (13-16); risco elevado (12 ou menos) ${ }^{(1)}$.

Para a avaliação do risco, os enfermeiros da instituição foram capacitados para a aplicação da escala, bem como a avaliação de surgimento de UP, seu estágio e local. Os dados são registrados pelos enfermeiros a cada 48 horas, em um programa informatizado. Isso permite emitir relatórios de gerenciamento com as informações referentes à permanência do paciente, o escore de risco, desenvolvimento de UP com local e estágio e medidas preventivas. Os dados utilizados no presente estudo são oriundos desses relatórios. Foram utilizadas as avaliações registradas pelos enfermeiros no momento da internação e da alta hospitalar.

As variáveis para caracterização demográfica analisadas foram: idade, sexo, dias de internação e especialidade. As variáveis relacionadas à UP foram os itens da escala de Braden e o escore nos dois momentos.

Os dados foram organizados em planilha Excel, exportados para o programa SPSS 11.5 (Statistc Package for Social Sciences2003) e analisados por estatística descritiva e inferencial, utilizando-se os testes Qui-quadrado e Teste $t$ de Student (não pareado e pareado). O projeto foi aprovado pelo Comitê de Ética em Pesquisa da Instituição sob registro 07/03910. Foi mantido sigilo quanto à identidade dos participantes. A equipe de pesquisa assinou um termo de responsabilidade de uso dos dados.

\section{APRESENTAÇÃO DOS RESULTADOS}

Dos 216 pacientes avaliados, 125 eram idosos, 34 adultos jovens e 54 adultos médios. A distribuição por sexo foi semelhante, sendo 109 homens (50,5\%) e 107 mulheres (49,5\%). A média de idade dos idosos foi de $73,16( \pm 7,8)$, não havendo diferença significativa entre mulheres e homens $(p=0,135)$. O maior percentual dos idosos era paciente de medicina interna $(25,6 \%)$,

Tabela 1 - Distribuição da amostra de pacientes idosos de acordo com o escore de risco para UP na primeira e na última avaliação, UTIGeral, jan-jul 2010.

\begin{tabular}{|l|cc|cc|}
\multicolumn{1}{|c|}{ Risco } & \multicolumn{3}{|c|}{ Primeira avaliação } & Última avaliação \\
Inexistente (21-23) & $\mathbf{n}$ & $\mathbf{\%}$ & $\mathbf{n}$ & $\%$ \\
\hline Baixo (17-20) & 7 & - & - & - \\
Moderado (13-16) & 17 & 13,6 & 4 & 11,4 \\
Elevado (12 e menos) & 101 & 80,8 & 25 & 71,4 \\
Total & 125 & 100,0 & 35 & 100,0 \\
\hline
\end{tabular}


seguida de nefrologia $(16,8 \%)$ e cirurgia geral (12\%). Quanto aos dias de internação, $42,4 \%$ dos idosos permaneceram na UTI por até 15 dias, $28 \%$ entre 16 e 30 dias e $29,6 \%$, 31 dias ou mais.

O risco para formação de UP na primeira e na última avaliação manteve-se elevado (tabela 1).

Comparando-se a média da primeira e da última avaliação dos pacientes (tabela 2), observou-se melhora dos escores na última avaliação $(p=0,000)$. Entretanto, ao considerar apenas os 35 idosos avaliados na primeira e última avaliação (amostra pareada), não houve alteração significativa no escore total ( $p=$ $0,319)$ e nas variáveis separadamente, a não ser no aspecto da nutrição $(p=0,000)$.

Considerando os fatores de risco de UP avaliados pela escala de Braden nos idosos (tabela 2), observou-se que, para o domínio da percepção sensorial, as médias dos subescores apresentaram-se entre os riscos totalmente limitado e muito limitado. Para o item atividade física, os pacientes obtiveram escore de risco indicando que os pacientes estavam acamados ou confinados à cadeira. A umidade da pele para os pacientes deste estudo, considerando a média, apresentou-se como ocasionalmente molhada. Os subescores indicaram pacientes totalmente imóveis ou com mobilidade bastante limitada. O item fricção e cisalhamento foi classificado como problema e potencial para a questão.

Houve diferença significativa entre os escores da última avaliação de Braden e dias de internação $(p=0,050)$. Observouse que a média do escore para os pacientes internados entre 16 e 30 dias foi a mais alta $(13,38)$.

As diferenças observadas entre o escore de Braden na primeira

avaliação e as especialidades não foram significativas $(p=0,452)$, assim como na última avaliação $(p=0,912)$. Entre as faixas etárias (tabela 3), não houve diferença significativa entre os escores das avaliações entre os adultos jovens e idosos, nem na primeira $(p=0,435)$, nem na última avaliação $(p=0,397)$, embora os escores dos idosos sejam mais altos. Entre adultos médios e idosos, não houve diferença significativa na primeira $(p=0,512)$ e na última avaliação $(p=0,136)$, e chama a atenção que os adultos médios tenham escores piores do que os idosos.

\section{DISCUSSÃO}

"Os pacientes da UTI utilizam diversas medicações que elevam o risco para UP. Sedativos e analgésicos prejudicam a mobilidade, os agentes hipotensores reduzem o fluxo

\section{sanguíneo e a} perfusão tissular"
Na UTI dessa instituição, assim como nos demais estudos nacionais pesquisados, a maioria dos pacientes internados era

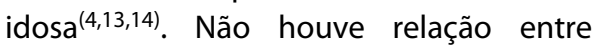
formação de UP e idade nessa investigação, o que também ocorreu em outro estudo(4). Deve-se levar em consideração que os pacientes da UTI utilizam diversas medicações que elevam o risco de UP. Sedativos e analgésicos prejudicam a mobilidade, os agentes hipotensores reduzem o fluxo sanguíneo e a perfusão tissular, tornando-os mais susceptíveis à pressão $(7,15,16)$. Isoladamente, a idade não poderia justificar o risco elevado para gênese de UP. Em estudos realizados em unidades de internação abertas, a idade avançada esteve relacionada à formação de UP(7,14). Em estudo realizado em um hospital-escola(15), a idade, associada a desnutrição, mobilidade e umidade, é um dos mais relevantes fatores envolvidos na fisiopatogênese das UP.

O tempo médio de permanência na UTI variou muito na literatura. Estudos realizados mostraram que, quanto maior
Tabela 2 - Distribuição da amostra de pacientes idosos de acordo com os itens da escala de Braden na primeira e na última avaliação, UTIGeral, jan-jul, 2010.

\begin{tabular}{|c|c|c|c|c|c|}
\hline $\begin{array}{c}\text { Item } \\
\text { avaliado }\end{array}$ & $\begin{array}{l}\text { Média - } \\
\text { primeira } \\
\text { avaliação } \\
(n=125)\end{array}$ & $\begin{array}{c}\text { Média - } \\
\text { primeira } \\
\text { avaliação } \\
(n=35)^{*}\end{array}$ & $\begin{array}{l}\text { Média - } \\
\text { última } \\
\text { avaliação } \\
(n=35)^{*}\end{array}$ & value** & $\begin{array}{c}\mathbf{P} \\
\text { value*** }\end{array}$ \\
\hline $\begin{array}{l}\text { Percepção } \\
\text { sensorial }\end{array}$ & 1,79 & 2,03 & 1,97 & 0,000 & 0,818 \\
\hline Atividade física & 1,09 & 1,09 & 1,11 & 0,000 & 0,571 \\
\hline $\begin{array}{l}\text { Umidade } \\
\text { da pele }\end{array}$ & 2,94 & 3,06 & 2,97 & 0,000 & 0,447 \\
\hline Mobilidade & 1,50 & 1,57 & 1,49 & 0,000 & 0,571 \\
\hline Nutrição & 1,86 & 1,57 & 2,37 & 0,000 & 0,000 \\
\hline Fricção & 1,22 & 1,29 & 1,23 & 0,000 & 0,324 \\
\hline Escore & 10,41 & 10,60 & 11,14 & 0,000 & 0,319 \\
\hline
\end{tabular}

Nota: * Dos 125 pacientes incluídos no estudo, apenas 35 tiveram avaliação na ocasião da alta da UTl; ** Teste-t não pareado; ${ }^{* * *}$ Teste-t pareado.
Tabela 3 - Escore da escala de Braden na primeira e na última avaliação para adultos jovens, médios e idosos, UTI geral, jan-jul, 2010.

\begin{tabular}{|c|c|c|c|c|c|}
\hline Avaliação & $\begin{array}{l}\text { faixa } \\
\text { etária }\end{array}$ & $n$ & Média & $\begin{array}{l}\text { Desvio- } \\
\text { padräo }\end{array}$ & $\mathbf{P}^{*}$ \\
\hline \multirow{3}{*}{$\begin{array}{l}\text { Primeira } \\
\text { avaliação }\end{array}$} & $\begin{array}{l}\text { adulto } \\
\text { jovem }\end{array}$ & 37 & 10,84 & 3,362 & 0,435 \\
\hline & $\begin{array}{l}\text { adulto } \\
\text { médio }\end{array}$ & 54 & 10,09 & 3,269 & 0,512 \\
\hline & $\begin{array}{l}\text { adulto } \\
\text { idoso }\end{array}$ & 125 & 10,41 & 2,794 & \\
\hline \multirow{3}{*}{$\begin{array}{l}\text { Última } \\
\text { avaliação }\end{array}$} & $\begin{array}{l}\text { adulto } \\
\text { jovem }\end{array}$ & 10 & 12,20 & 4,367 & 0,397 \\
\hline & $\begin{array}{l}\text { adulto } \\
\text { médio }\end{array}$ & 19 & 9,79 & 3,102 & 0,136 \\
\hline & $\begin{array}{l}\text { adulto } \\
\text { idoso }\end{array}$ & 35 & 11,14 & 3,154 & \\
\hline
\end{tabular}

*Idoso em relação ao adulto jovem e médio. 
o tempo de internação, maior o número de pacientes com formação de UP(4,14). Nesta investigação, o escore da escala de Braden obteve maior concentração nos escores de risco elevado e moderado para ocorrência de UP nas duas avaliações, semelhante a outros estudos ${ }^{(13)}$.

A percepção sensorial muito limitada, como a dos idosos deste estudo, determina a não-percepção do desconforto e, consequentemente, a pessoa não realiza mudanças de posição para aliviar essa pressão, dependendo dos cuidados de enfermagem ${ }^{(4)}$. O excesso de umidade da pele, verificada nos resultados deste estudo, por sudorese, incontinência urinária ou fecal, pode tornála mais suscetível à maceração. A instalação de sondagem vesical de demora para balanço hídrico e tratamento da incontinência ou retenção urinária influenciou nos resultados da pesquisa, mantendo os pacientes com menor umidade da pele $\mathrm{e}^{(4)}$.

A imobilidade do paciente é tida como principal fator para ocorrência de UP. Outro estudo ${ }^{(14)}$ mostrou que os pacientes que apresentaram UP eram os que tinham maior número de dias de imobilidade.

A avaliação da nutrição é uma limitação da escala de Braden, visto que ela avalia apenas a ingestão e não o estado nutricional. A má nutrição contribui para a formação de UP por diminuir a tolerância tissular à pressão. $\mathrm{O}$ estado nutricional dos pacientes críticos geralmente é alterado por jejum prolongado, estados patológicos e hipercatabólicos, cirurgias e desnutrição(3). A melhora do fator nutricional dos pacientes em estudo pode ter sido influenciada pela instalação de sonda nasoenteral.

Outros fatores avaliados pela escala de Braden são fricção e cisalhamento, que podem estar relacionados com a diminuição da percepção sensorial e da mobilidade encontrados em nossos resultados.

\section{CONCLUSÕES}

A avaliação dos fatores de risco para formação de UP na UTI aponta que não houve diferença de risco entre os pacientes idosos e os adultos jovens e médios. A maior parte dos pacientes, independentemente da idade, possui risco elevado ou moderado para ocorrência de úlcera de pressão. Portanto, é de suma importância que os enfermeiros estejam motivados para aplicar a escala de Braden e que sensibilizem e capacitem sua equipe para a adoção de intervenções de prevenção.

A realização de estudos de prevalência e incidência de úlcera de pressão nessa unidade, com delineamentos longitudinais, seria interessante, a fim de verificar se as medidas terapêuticas preconizadas pelo Protocolo de Prevenção de UP estão sendo eficazes para sua prevenção.

\section{Referências}

1. Harada MJCS. O erro humano e a segurança do paciente. São Paulo: Atheneu; 2006.

2. D'Inocenzo M. Indicadores, auditorias, certificaçōes: ferramentas de qualidade para a gestão em saúde. São Paulo: Martinari; 2006.

3. NPUAP. Pressure ulcer stages - pressure ulcer definition National Pressure Ulcer Advisory Panel, 2009 [Internet]. [citado em 2010 No 08]. Disponível em: http://www.npuap.org/pr2.htm.

4. Fernandes LM, Caliri MHL. Uso da Escala de Braden e de Glasgow para identificação do risco para úlceras de pressão em pacientes internados em centro de terapia intensiva [Internet]. [citado em 2010 Nov 09]. Disponível em: http://www.scielo.br/scielo.php?script=sci_arttext\&pid=S01041169200800060 0006\&lng=en.

5. Caromano FA, Jung TC. Estudo comparativo do desempenho em testes de força muscular entre indivíduos jovens e idosos através da miometria. Rev Fisioter. 1999;6:101-12.

6. Ramos LR. Fatores determinantes do envelhecimento saudável em idosos residentes em centro urbano: Projeto Epidoso, São Paulo [Internet]. [citado em 2010 Nov 09]. Disponível em: http://www.scielo.br/scielo.php?script=sci_ arttext\&pid=S0102-311X2003000300011\&lng=en

7. Moro A, Maurici A, Valle JB, Zaclikevis VR, Kleinubing JH. Avaliação dos pacientes portadores de lesão por pressão internados em hospital geral [Internet]. [citado em 2010 Nov 09]. Disponível em: http://www.scielo.br/scielo. php?script=sci_arttext\&pid=S010442302007000400013\&lng=en. 8. Elizabete VF. Tratado de geriatria e gerontologia. $2^{\mathrm{a}}$ ed. Rio de Janeiro:
Guanabara Koogan; 2006.

9. Fernandes LM. Úlcera de pressão em pacientes críticos hospitalizados: uma revisão integrativa da literatura [dissertação]. Ribeirão Preto: Escola de Enfermagem de Ribeirão Preto da Universidade de São Paulo; 2000.

10. Stephen H. Delineando a pesquisa clínica: uma abordagem epidemiológica. $3^{\text {a }}$ ed. Porto Alegre: Artmed; 2008.

11. Papalia DE. Desenvolvimento humano. $8^{\mathrm{a}}$ ed. Porto Alegre: Artmed; 2006.

12. Costa IG. Incidência de úlcera de pressão e fatores de risco relacionados em pacientes de um Centro de Terapia Intensiva [dissertação]. São Paulo: Escola de Enfermagem de Ribeirão Preto da Universidade de São Paulo; 2003.

13. Cremasco MF. Úlcera por pressão: risco e gravidade do paciente e carga de trabalho de enfermagem. Acta Paul Enferm. 2009;22.

14. Louro M, Ferreira M, Póvoa P. Avaliação de protocolo de prevenção e tratamento de úlceras de pressão [Internet]. [citado em 2010 Nov 25]. Disponível em: http://www.scielo.br/scielo.php?script=sci_arttext\&pid=\$01035 07X2007000300012\&lng=en.

15. Rogenski NMB, Santos VLCG. Estudo sobre a incidência de úlceras por pressão em um hospital universitário [Internet]. [citado em 2010 Nov 25]. Disponível em: http://www.scielo.br/scielo.php?script=sci_arttext\&pid=S01041 $1692005000400003 \& \operatorname{lng}=$ en.

16. Blanes L, Duarte IS, Calil JA, Ferreira LM. Avaliação clínica e pidemiológica das úlceras por pressão em pacientes internados no Hospital São Paulo [Internet]. [citado 2010 Nov 25].Disponível em: http://www.scielo.br/scielo. php?script=sci_arttext\&pid=S0104-42302004000200036\&lng=en. 